Jeffrey M. Zink

Rita Singh-Parikshak

C. Starck Johnson

David N. Zacks

\section{Hypopyon uveitis associated with systemic lupus erythematosus and antiphospholipid antibody syndrome}

Received: 14 May 2004

Revised: 16 July 2004

Accepted: 12 August 2004

Published online: 13 October 2004

(C) Springer-Verlag 2004

J. M. Zink ( ) R. Singh-Parikshak ·

C. S. Johnson - D. N. Zacks

Retina Service, Kellogg Eye Center,

University of Michigan,

1000 Wall St., Ann Arbor, MI 48105, USA

e-mail: jzink@med.umich.edu

Tel.: +1-734-7637711

Fax: +1-734-9362340

\begin{abstract}
Purpose: To report a case of hypopyon uveitis associated with systemic lupus erythematosus and antiphospholipid antibody syndrome. Methods: Interventional case report: a 49-year-old woman with a history of systemic lupus erythematosus and antiphospholipid antibody syndrome presented with sudden onset of pain, redness, photophobia, and decreased vision in the left eye. Examination revealed hypopyon uveitis and vasoocclusive retinopathy. Results: Fol-
\end{abstract}

lowing treatment with intravenous steroids and cyclophosphamide, the patient's vision improved from $\mathrm{CF}$ to $20 / 80$ with no inflammation 6 months following initial presentation. Conclusions: In this case, systemic lupus and antiphospholipid syndrome were associated with hypopyon uveitis. Prompt treatment with systemic immunosuppressive therapy resulted in improvement in the hypopyon uveitis and vaso-occlusive retinopathy.

\section{Introduction}

Systemic lupus erythematosus is commonly associated with a microangiopathic retinopathy characterized by intraretinal hemorrhages and cotton-wool spots. Occasionally, SLE and antiphospholipid antibody syndrome are associated with a severe vaso-occlusive retinopathy or choroidopathy $[3,4,6]$. To our knowledge, hypopyon uveitis has not been reported in systemic lupus erythematosus or antiphospholipid antibody syndrome.

\section{Case report}

A 49-year-old woman was referred to the Retina Service of the Kellogg Eye Center. She had a 26-year history of systemic lupus complicated by recurrent episodes of lower extremity vasculitis, antiphospholipid antibody syndrome, polyarthralgias, and plaquenil-associated maculopathy. The patient complained of progressive pain, redness, photophobia, and decreased vision in the left eye. One week prior to presentation the patient had been hospitalized for a lupus flare characterized by polyarthralgias and lower extremity vasculitis that was treated with pulse intravenous steroids. The patient's medications on discharge, 3 days prior to presentation, included oral prednisone and azathioprine. On examination, bestcorrected visual acuity was 20/60 OD and CF at 6 in. $(15 \mathrm{~cm}) \mathrm{OS}$.
The right eye was significant only for her baseline plaquenil-associated maculopathy. The left eye, however, showed moderate conjunctival injection and chemosis with $3+$ cells in the anterior chamber and a layered hypopyon (Fig. 1a). The iris vessels were sheathed with exudates and intrastromal hemorrhages (Fig. 1b). Dilated retinal examination showed $3+$ vitreous cells and $360^{\circ}$ of intraretinal hemorrhages, retinal edema, cotton-wool spots, and arterial and venous sheathing (Fig. 2a). Fluorescein angiography showed blockage of the choroidal fluorescence in the areas of the retinal hemorrhages, severe capillary dropout, and late leakage from inflamed vessels (Fig. 2b,c).

The patient was admitted to the hospital and started on intravenous methylprednisolone and aggressive topical steroid therapy. Since hypopyon uveitis has not been reported to be associated with systemic lupus erythematosus or antiphospholipid antibody syndrome, the diagnosis of Behcet's disease was considered. Behcet's disease can be associated with antiphospholipid syndrome and is characterized by hypopyon uveitis and occlusive vasculitis [1]. Our patient was negative for HLA B5 and did not meet the International Study Group criteria for Behcet's disease. This suggested that the hypopyon uveitis was a novel ocular manifestation of systemic lupus. Magnetic resonance imaging of the brain did not reveal any evidence of CNS vasculitis. In consultation with her rheumatologist, the patient was also started on intravenous cyclophosphamide. One week following presentation the hypopyon had resolved. The patient was discharged and received continued treatment with monthly intravenous cyclophosphamide infusions. She tolerated a slow taper of the oral and topical steroids. By 6 months her visual 
Fig. 1 a anterior segment color photograph showing layered hypopyon and intrastromal iris hemorrhages. b higher magnification view of sheathed iris vessels and intrastromal hemorrhages
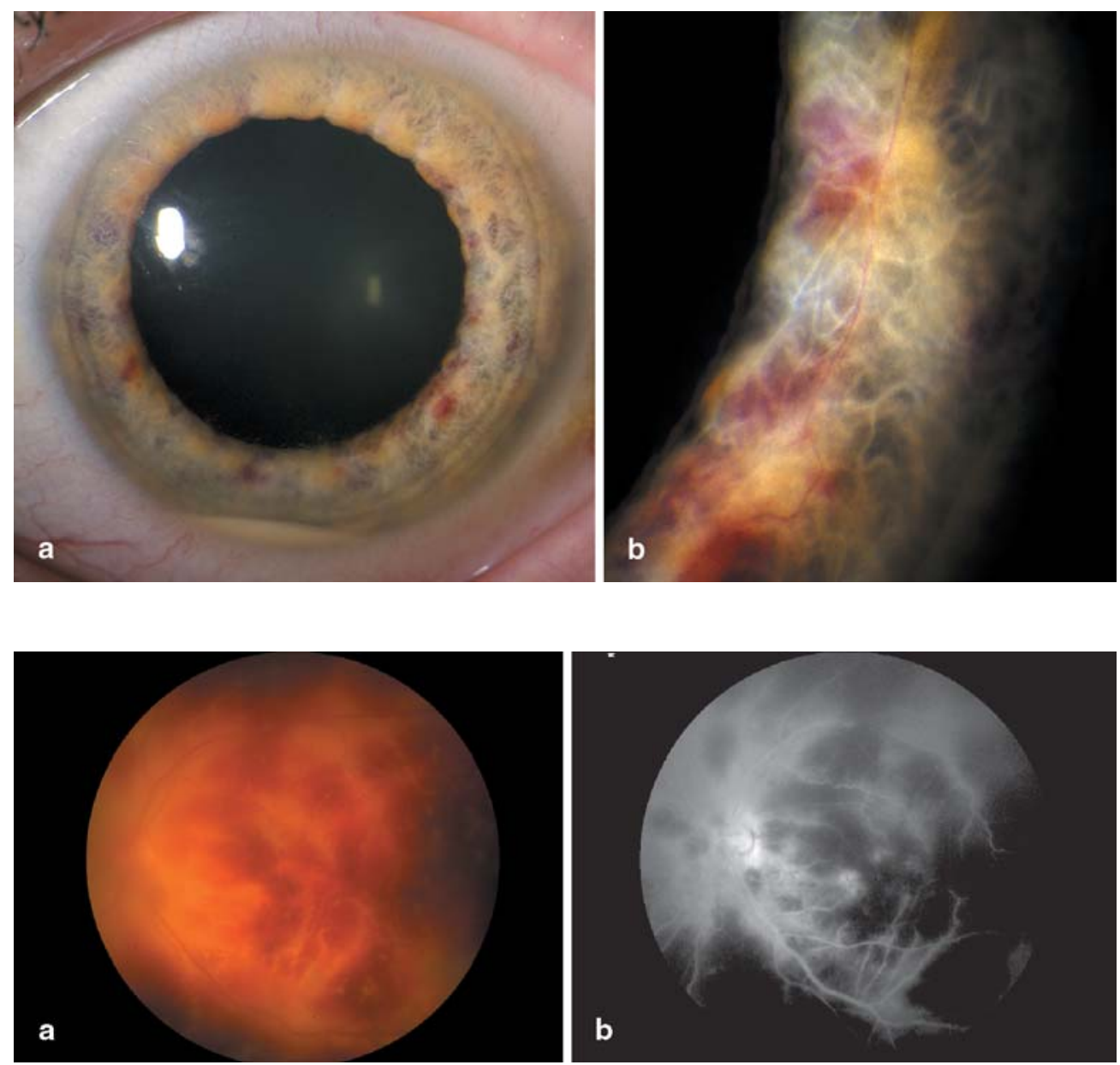

Fig. 2 a Color fundus photo showing intraretinal hemorrhages, edema, cotton-wool spots, and arterial and venous sheathing. b Late-phase fluorescein angiograph showing severe capillary dropout and late leakage from inflamed vessels acuity had improved to her baseline of 20/80 without recurrence of any intraocular inflammation.

\section{Discussion}

Vaso-occlusive retinopathy has been reported in patients with systemic lupus erythematosus and antiphospholipid antibody syndrome $[4,6]$. In addition to retinopathy, lupus has been shown to be associated with uveitis. The proportion of the uveitis patients referred to a tertiary care center over a 10-year period who had a diagnosis of lupus was $4.8 \%$ [5]. Anticardiolipin antibodies are also more commonly associated with occlusive retinopathy; however, there are reports of iritis, scleritis, filamentary keratitis, and vitritis [2].

Given the novel finding of hypopyon uveitis in this case, it is important to establish that the patient met the diagnostic criteria for systemic lupus erythematosus. The patient had a 26-year history of systemic lupus and was followed up closely by our rheumatology clinic. Her lupus was characterized by joint involvement, possible previous CNS vasculitis, glomerulonephritis with chronic renal failure, positive ANA, positive antiRo and antidsDNA antibody, persistent lymphopenia, and low $\mathrm{C} 3$ and $\mathrm{C} 4$ levels. The patient also had a history of antiphospholipid antibody syndrome characterized by a markedly raised DRVVT with mixing studies positive for coagulation protein inhibitor. In addition, the patient test-ed positive for IgM anticardiolipin antibody and IgM antibeta2GPI. The presence of antiphospholipid anti- body syndrome was further supported by a history of chronic thrombocytopenia, false-positive VDRL, cognitive dysfunction, mitral valve regurgitation consistent with Libman-Sachs endocarditis, and deep venous throm-bosis. These findings confirmed that the patient met the clinical diagnostic criteria for lupus and strongly supported the diagnosis of antiphospholipid antibody syndrome. Furthermore, our patient did not meet the In- ternational Study Group diagnostic criteria for Behcet's disease.

To our knowledge, this is the first reported case of hypopyon uveitis seen in the setting of systemic lupus erythematosus or antiphospholipid antibody syndrome. This case demonstrates the importance of prompt systemic immunosuppressive therapy in controlling the ocular manifestations of lupus. 


\section{References}

1. Famularo G, Antonelli S, Barracchini A et al (2002) Catastrophic antiphospholipid syndrome in a patient with Behcet's disease. Scand J Rheumatol 31:100-102

2. Miserocchi E, Baltatzis S, Foster CS (2002) Ocular features associated with anticardiolipin antibodies; a descriptive study. Am J Ophthalmol 131:451-456
3. Nguyen QD, Uy HS, Akpek EK et al (2000) Choroidopathy of systemic lupus erythematosus. Lupus 9:288-298

4. Read RW, Chong LP, Narsing RA (2000) Occlusive retinal vasculitis associated with systemic lupus erythematosus. Arch Ophthalmol 118:588589
5. Rodriguez A, Calonge M, PedrozaSeres M et al (1996) Referral patterns of uveitis in a tertiary eye care center. Arch Ophthalmol 114:593-599

6. Snyers B, Lambert M, Hardy JP (1990) Retinal and choroidal vaso-occlusive disease in systemic lupus erythematous associated with antiphospholipid antibodies. Retina 10:255-260 